\title{
NS-Raubgutforschung an der Württembergischen Landesbibliothek
}

\section{Das Projekt}

Im Projekt "Suche nach NS-Raubgut in den nach 1944 antiquarisch wiederbeschafften Beständen der Württembergischen Landesbibliothek" werden seit Juni 2016 Teile der Bibliotheksbestände nach NS-Raubgut untersucht. Finanziert werden die bis Mai 2019 dauernden Arbeiten zu gleichen Teilen von der "Stiftung Deutsches Zentrum Kulturgutverluste" und dem Ministerium für Wissenschaft, Forschung und Kunst Baden-Württemberg.

In der Zeit des Nationalsozialismus wurden von staatlicher Seite nicht nur massenhaft Kunstwerke geraubt. In noch viel größerem Maßstab betraf die staatliche Enteignung Bücher, von besonders wertvollen Exemplaren bis hin zu einfacher Gebrauchsliteratur. Ganze Bibliotheken wurden geplündert, die Bücher zerstreut, vernichtet oder wiederum von öffentlichen Bibliotheken vereinnahmt.

Die NS-Raubgutforschung an Bibliotheken beschäftigt sich damit, Bücher, die ihren Vorbesitzern geraubt wurden und die bis heute größtenteils unbemerkt in vielen Bibliotheksmagazinen liegen, aufzuspüren und zu identifizieren. Sie ist damit ein Zweig der Provenienzforschung, die sich mit der Herkunft der Bücher in Bibliotheken, also ihrer Provenienz, allgemein beschäftigt.

Da oftmals eindeutige Beweise für den Bücherraub fehlen, müssen in der NS-Raubgutforschung mühsam Spuren zusammengetragen werden, zum einen aus Quellen zum Buchzugang der damaligen Zeit, die in den Bibliotheken noch vorhanden sind, zum anderen aus den Büchern selbst, die oftmals Besitzeinträge enthalten, die Aufschluss über Vorbesitzer ${ }^{1}$ geben können.

Auch wenn Institutionen aufgrund der Verjährungsfristen heute rechtlich nicht mehr verpflichtet sind, NS-Raubgut zurückzuerstatten, haben sich die Bundesrepublik Deutschland, die Bundesländer sowie die kommunalen Spitzenverbände nach der so genannten "Washingtoner Erklärung" vom

\section{Dezember 1998 dazu verpflichtet, während} der NS-Zeit geraubtes Kulturgut zu identifizieren, dessen ehemalige Eigentümer oder deren Erben ausfindig zu machen und "gerechte und faire Lösungen" zu finden.

Die NS-Raubgutforschung in Bibliotheken hat demnach die Aufgabe, geraubte Bücher ausfindig zu machen, zu dokumentieren und - wenn irgend möglich - den Opfern oder ihren Erben zurückzuerstatten. Diese "Restitution" an die Opfer oder ihre Erben ist das oberste, wenn auch nur selten erreichbare Ziel der NS-Raubgutforschung.

Um die Vorgehensweise bei der Suche nach NSRaubgut an der Württembergischen Landesbibliothek besser zu verstehen, lohnt ein kurzer Blick in die Bibliotheksgeschichte. Das Gebäude der Bibliothek wurde 1944 bei einem Luftangriff weitgehend zerstört, darunter insbesondere auch die Buchbestände, die ab 1933 ins Haus gekommen waren. Damit muss auch eventuell vorhandenes NS-Raubgut zumindest zum größten Teil zerstört worden sein, es sei denn, es handelte sich um geraubte Bände, die in den sachlich aufgestellten Altbestand der Bibliothek eingeordnet worden waren.

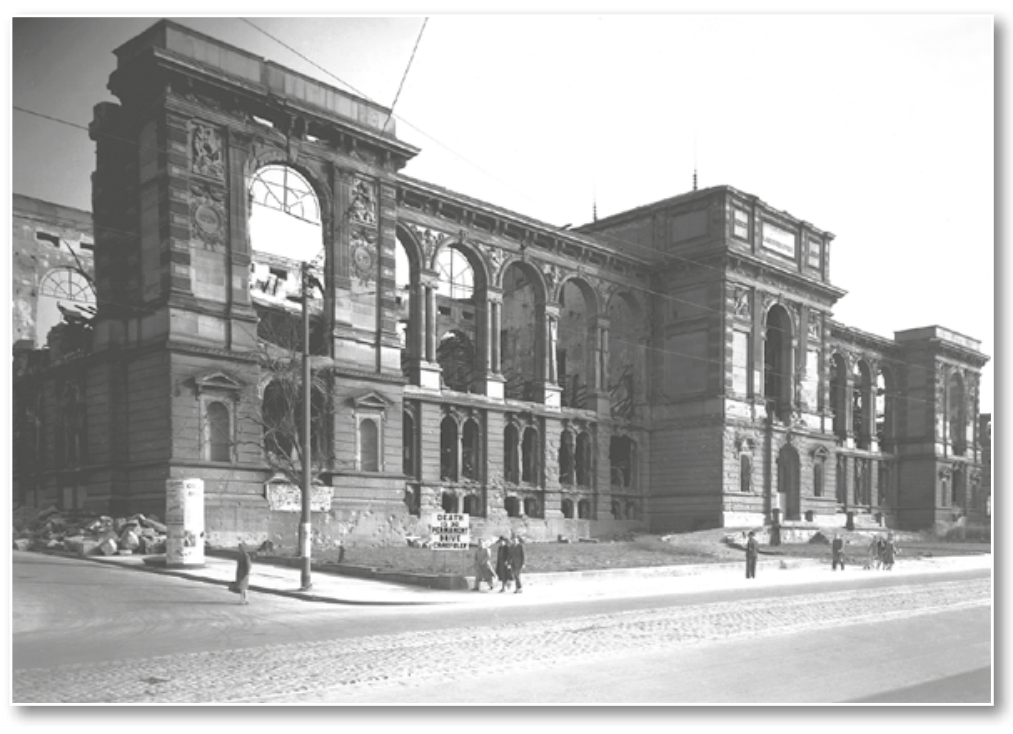

Abb. 1: Das weitgehend zerstörte Gebäude der Württembergischen Landesbibliothek nach 1944 
Gleich nach der Zerstörung großer Teile des Bibliotheksbestands begann man jedoch mit der Wiederbeschaffung der verlorenen Bücher, zumeist auf dem antiquarischen Markt, der zu dieser Zeit aber wohl zu einem großen Teil aus geraubten Büchern bestand. Die Suche nach NS-Raubgut an der Württembergischen Landesbibliothek nimmt gezielt diese antiquarisch wiederbeschafften Bestände in den Blick. Gefahndet wird also nach so genanntem "Raubgut aus zweiter Hand“. Für diese antiquarisch erworbenen Bestände führte man an der Württembergischen Landesbibliothek 1950 ein besonderes Signaturensegment ein. Diese Signatur beginnt mit dem Großbuchstaben „A", dem eine Ziffer für das entsprechende Erwerbungsjahr folgt. Der so genannte "Jahresring" A1 enthält also Bücher, die 1950 antiquarisch erworben wurden. Der zweite Teil der Signatur - abgetrennt durch einen Schrägstrich - besteht schlicht aus einer fortlaufenden Nummer - lateinisch nummerus currens. Das Buch mit der Signatur "A $1 / 1$ " ist also das erste antiquarisch erworbene Buch, das im Jahr 1950 ins Haus kam. Entsprechend ihrer Signatur sind die Bände im Magazin fortlaufend geschlossen aufgestellt. Somit eignen sich diese Bestände sehr gut für die Durchsicht am Regal. Im Projekt an der WLB sollen sämtliche antiquarischen Erwerbungen der Jahre 1950 bis ca. 1970 - die „Jahresringe“ A1 bis A20 - in die Untersuchung mit einbezogen werden, insgesamt ca. 60.000 Bände.

\section{Vorgehensweise}

Der oben beschriebene Bestand wird im Rahmen des Projekts systematisch Band für Band (autoptisch) durchgesehen. Die Bücher werden dabei auf Spuren von Vorbesitzern hin durchsucht. Solche Spuren sind vor allen Dingen Besitzmerkmale wie Exlibris, handschriftliche Eintragungen, Widmungen oder Stempel. Alle diese Merkmale lassen sich zusammenfassend als "Provenienzmerkmale" bezeichnen.

Die gefundenen Merkmale werden in einer hauseigenen allegro-Datenbank verzeichnet. Ein Daten-

sfiste

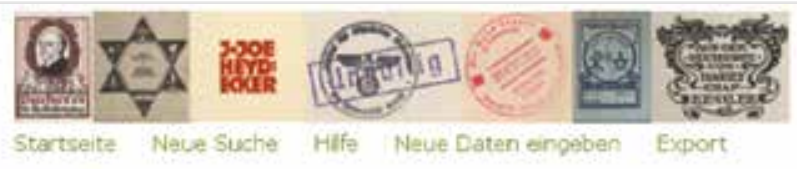

Startseite Neve Suche Hilfe Neve Daten einpeben Export Sundestbiliogthet stuttgart rschung

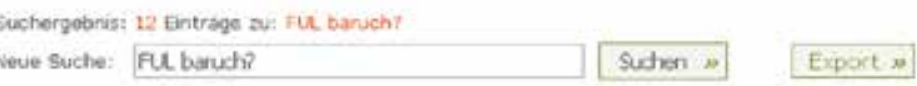

F. Provenenz arain pay

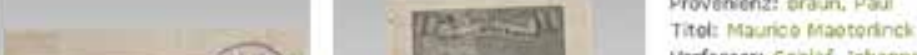

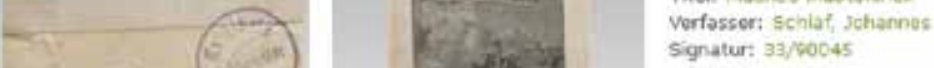

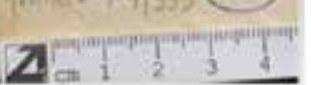
cation 120

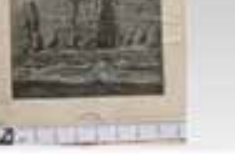

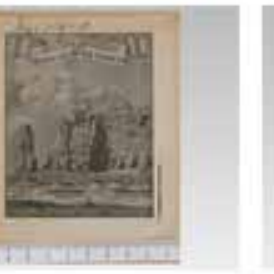

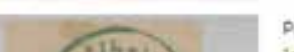
Prowenienz: Baruch ceorg; Baruch: Titel: Destieu von Lilenction gesarmatie worke Verfasecr: Donmel, fiehard (itg.? Signatur: $A 5 / 316-1$

Abb. 2: Beispiel für eine Ergebnisliste in der hauseigenen Datenbank zur NS-Raubgutforschung

satz umfasst dabei sowohl Angaben zum Buch als auch Angaben zum Besitzmerkmal. Hinzu kommen Scans der entsprechenden Merkmale.

Auf der Basis dieser Daten werden erste Recherchen nach den jeweiligen Personen oder Institutionen, auf die die Provenienzmerkmale verweisen, vorgenommen. Gefragt wird dabei, ob es irgendeinen Hinweis darauf gibt, dass es sich um NSVerfolgte handeln könnte und die Bücher eventuell unrechtmäßig enteignet worden sein könnten. Bei Institutionen kommen hier beispielsweise jüdische Gemeinden, Freimaurerlogen, Arbeitervereinigungen oder demokratische Parteien in Frage. Aber auch Hinweise auf NS-Organisationen, also auf „Täterorganisationen", müssen berücksichtigt werden, denn auch diejenigen, die aktiv geraubt haben, können ihre Spuren in den Büchern hinterlassen haben, wie zum Beispiel die Gestapo, die zahlreiche Bücher beschlagnahmt hat.

Verfolgte Privatpersonen waren in erster Linie die nach den Nürnberger Gesetzen als „Juden“ geltenden Deutschen, aber auch Widerstandskämpfer, Mitglieder demokratischer Parteien, Gewerkschaftler, Freimaurer und andere. 
Die entdeckten Provenienzmerkmalen in den Büchern reichen vielfach nicht aus, um genaue Rückschlüsse auf individuelle Personen oder Institutionen ziehen zu können. Es gibt dementsprechend große Unterschiede, wie genau sich aus diesen Merkmalen nachweisen lässt, ob es sich bei einem Exemplar wirklich um ehemaligen Besitz von NS-Verfolgten handelt. Noch schwieriger wird es nachzuweisen, ob ein entsprechendes Buch also um NS-Raubgut handelt.

In den meisten Fällen wird man daher nur einen mehr oder weniger großen Verdacht auf NS-Raubgut ermitteln können. Dementsprechend werden die jeweiligen Bücher anhand ihrer Provenienzmerkmale in verschiedene Kategorien eingeordnet, je nachdem wie wahrscheinlich es ist, dass es sich um NS-Raubgut handelt. Diese reichen von Kategorie 1 "geklärt, kein Raubgut" über „unspezifisch", "verdächtig" bis hin zu „NS-Raubgut" oder eben "restituiert".

\section{Transparenz}

Um die Ergebnisse der Untersuchung möglichst transparent zu machen, werden im Projekt an der WLB Provenienzmerkmale, die einen Verdacht auf NS-Raubgut nahelegen, im Provenienz-Wiki des Gemeinsamen Bibliotheksverbundes (GBV) verzeichtatsächlich unrechtmäßig enteignet wurde, es sich

Die Provenienzmerkmale einzelner Bücher werden darüber hinaus auch im Bibliothekskatalog nachgewiesen. Sie werden im Online-Katalog des Südwestdeutschen Bibliotheksverbundes (SWB) (http:// swb.bsz-bw.de/) verzeichnet und diese Daten dann in den Online-Katalog der WLB übernommen. Diese verdächtigen Bücher werden zudem für die Benutzung gesperrt. Die Hinweise in den Bibliothekskatalogen verweisen direkt auf die Opfer des Bücherraubs, sie bilden in den Katalogen gleichermaßen virtuelle "Stolpersteine" analog zu den realen "Stolpersteinen", die in vielen Städten von entsprechenden Initiativen vor den Gebäuden, in denen die Opfer zuletzt gelebt haben, verlegt werden und die direkt vor Ort an die Opfer erinnern sollen.

\section{Ziel der NS-Raubgutforschung in Bibliotheken ist} es, wie oben schon erläutert, den Opfern oder ihren Erben, die zu Unrecht geraubten Bücher zurückzuerstatten. Aus anderen Bibliotheken sind Fälle bekannt, in denen NS-Raubgut-Bücher aus Bibliotheken an Erben zurückgegeben wurden, die nun mit diesen Büchern die einzigen erhaltenen Gegenstände aus dem Besitz ihrer Vorfahren in den Händen halten. In den allermeisten Fällen geht es dabei auch nicht um materielle Werte - wie zum Beispiel häufig bei der NS-Raubgutforschung im Bereich der Kunstmuseen - sondern vielmehr um ideelle Werte. Die Suche nach Opfern oder den erbberechtigten Verwandten stellt sich häufig als äußerst schwierig dar. Langwierige Recherchen im Internet und in Archiven sind dazu nötig und führen nicht immer zu einem Ergebnis. An der WLB konnten daher bis jetzt auch nur erste Schritte in diesem Bereich getan werden.

Im Laufe des Projekts sollen Bücher mit verdächtigen Provenienzmerkmalen auch noch in der Datenbank Lost Art (http://www.lostart.de/Webs/DE/Datenbank/Index.html) der Stiftung Deutsches Abb. 3: Beispiel für einen Eintrag der WLB im „Provenienz-Wiki” des GBV

net (http://provenienz.gbv.de/Hauptseite), um so die Funde öffentlich zu machen und den Austausch mit anderen Provenienzforschern zu erleichtern. Insgesamt wurden während der bisherigen Projektdauer 41 Sätze in dieser Datenbank angelegt.
Zentrum Kulturgutverluste verzeichnet werden. Sie werden hier als „Fundmeldungen“ veröffentlicht, insbesondere in Fällen, in denen ein unrechtmäBiger Entzug wahrscheinlich oder erwiesen ist, jedoch noch keine konkreten Opfer oder deren Erben ermittelt werden konnten. 


\section{Erste Ergebnisse}

In den ersten acht Monaten des Projekts an der Württembergischen Landesbibliothek sind insgesamt schon über 23.000 Bände durchgesehen worden. In ca. 8.000 Bänden wurden Provenienzmerkmale gefunden. Da es unter innen zahlreiche mehrfach vorkommende Merkmale gibt, wurden nicht alle davon auch gescannt. Dennoch liegt die Anzahl der gescannten Merkmale recht hoch bei über 3.800. Über 2.500 Bände wurden bis jetzt in die hauseigene Datenbank aufgenommen. Da in zahlreichen Bänden mehrere Provenienzmerkmale vorkommen - zum Beispiel ein Exlibris und eine handschriftliche Widmung - übersteigt die Zahl der gescannten Merkmale die Zahl der in die Datenbank aufgenommenen Bücher. Unter den in der Datenbank verzeichneten Bänden können bei der Mehrzahl ausgehend von den Besitzeinträgen keine oder nur zumindest keine eindeutigen Schlüsse auf die Vorbesitzer gezogen werden: Viele handschriftliche Einträge sind unleserlich, bei vielen Namen kann die Identität nicht eindeutig geklärt werden, und zu vielen der gefundenen Personen oder Institutionen liegen keine ausreichenden Informationen vor, um beurteilen zu können, ob sie Verfolgte des NS-Staates waren oder nicht. Bei immerhin 444 Bänden besteht jedoch ein Verdacht auf NS-Raubgut, dem im weiteren Verlauf des Projekts noch weiter nachgegangen werden wird. Die im folgenden angeführten Beispiele dieser Verdachtsfällen sollen einen kurzen Einblick in die Vielfalt der bisher in der Württembergischen Landesbibliothek gefundenen Hinweise auf NSRaubgut geben.

Einige der gefundenen Besitzmerkmale stammen von Institutionen, und zwar sowohl von Täter- als auch von Opferinstitutionen. Zu den Täterorganisationen zählen staatliche oder NSDAP-Institutionen, die im Verdacht stehen, an Raubgutaktionen beteiligt gewesen zu sein. Zu den Opferorganisationen zählen jeweils Einrichtungen, die vom NS-Staat verfolgt wurden: Dies können jüdische Gemeinden, Freimaurerlogen, Parteien, Gewerkschaften oder andere sein. Zu den verfolgten Privatpersonen sind Juden, Freimaurer, Gewerkschaftler sowie Oppositionelle und Widerstandskämpfer zu zählen.
Da es sich in den meisten Fällen wohl um „Raubgut aus zweiter Hand" handeln dürfte, ist es nicht überraschend, dass die gefundenen Besitzmerkmale von Personen und Institutionen aus dem ge-
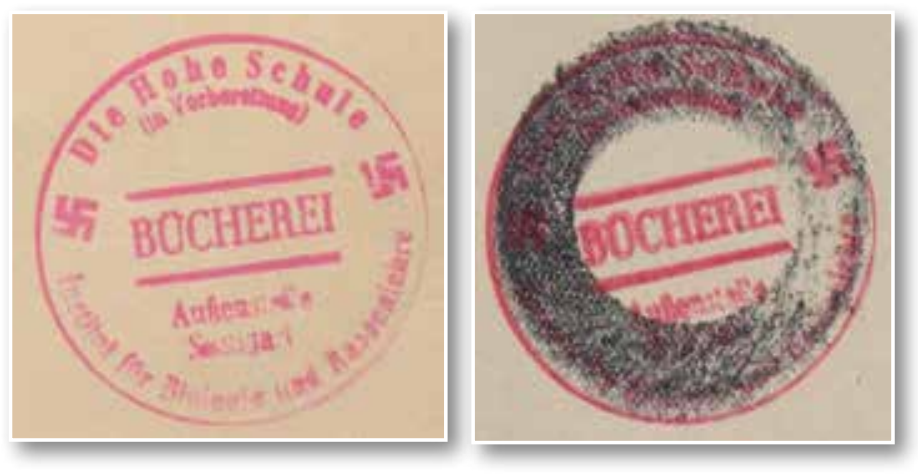

Abb. 4 und 5: Stempel der "Hohen Schule. Außenstelle Stuttgart", z.T. unkenntlich gemacht (in: A4a/250 und A1/617)

samten Gebiet des damaligen Deutschen Reiches und darüber hinaus stammen und nicht nur aus Stuttgart und Umgebung oder aus Württemberg. Das am häufigsten im untersuchten Bestand vorkommende verdächtige Provenienzmerkmal ist der Besitzstempel der so genannten "Hohen Schule". Diese war als Parteihochschule der NSDAP geplant und sollte verschiedene Außenstellen umfassen. Die gesamte Einrichtung befand sich jedoch zunächst noch „in Vorbereitung". Eine der Außenstellen, das so genannte "Institut für Biologie und Rassenkunde", wurde in Stuttgart aufgebaut.

Der entsprechende Stempel der "Hohen Schule (in Vorbereitung) Außenstelle Stuttgart. Institut für Biologie und Rassenkunde" (so der Wortlaut) fand sich bisher in insgesamt 242 Bänden. Es ist davon auszugehen, dass dieses Institut wie viele andere neu gegründete NS-Institutionen beim Aufbau seiner Bibliothek auch von NS-Raubgut profitiert hat. In einzelnen Büchern ließen sich weitere Besitzmerkmale von Personen finden, die den Verdacht auf Raubgut begründen können. Über das Institut in Stuttgart im Allgemeinen und zum Aufbau der Bibliothek im Speziellen konnten bisher weder aus der Forschungsliteratur noch aus Archivmaterial Erkenntnisse gewonnen werden. In Unterlagen aus dem Archiv der Landesbibliothek ist jedoch erwähnt, dass die Bibliothek des Instituts der "Hohen Schule" in der Kriegszeit nach Schelklingen ausgelagert worden war. Im Mai 1946 wurden die Bücher in die Landesbibliothek gebracht und offensichtlich im Laufe der folgenden Jahre in den eigenen Bestand eingearbeitet. 
Eine weitere NS-Organisation, deren Buchbestände zum großen Teil aus Raubgut stammen dürften, war das so genannte „Institut für Staatsforschung" mit Sitz in Berlin. Stempel dieses Instituts fanden sich bis jetzt in insgesamt drei Büchern aus dem untersuchten Bestand. Entsprechende Funde sind auch aus anderen Bibliotheken bekannt.

Auch Bände, die einstmals Freimaurerlogen gehörten, kommen in dem bisher untersuchten Bestand vor. Freimaurer wurden vom NS-Regime verfolgt, sämtliche Logen 1935 verboten. Auch in zahlreichen anderen Bibliotheken, die bisher Bestände auf NS-Raubgut hin untersucht haben, sind Bände aus Freimaurer-Bibliotheken gefunden worden. Nur ein Exemplar konnte bis jetzt mit dem Besitzeintrag einer jüdischen Institution gefunden werden. Dabei handelt es sich um einen Stempel des "Israelitischen Gemeinde-Vorsteher-Amtes Stuttgart". Auf welchem Weg dieser Band in die Bibliothek gekommen ist, ist noch unklar. In drei Bänden wurde der Stempel „Karl Marx Haus Trier" gefunden. Neben Juden und Freimaurern galten den Nationalsozialisten "Marxisten" als Hauptgegner, die auch die radikalsten Verfolgungsmaßnahmen erleiden mussten. Etliche wurden ermordet. In Trier wurde die Eröffnung des geplanten Museums im Karl-Marx-Haus durch die nationalsozialistische "Machtergreifung" im Januar 1933 verhindert, die Museumsbibliothek enthielt jedoch offenbar schon annähernd 3.000 Bände. Im März 1933 wurde das Gebäude von SA und SS besetzt. Die meisten Einrichtungsgegenstände und Ausstellungsobjekte sowie die Bücher gelten heute als verschollen. Solche Hinweise aus der Geschichte einer Einrichtung können schon als ausreichend angesehen werden, um die Restitution an etwaige Nachfolgeinstitutionen zu rechtfertigen.

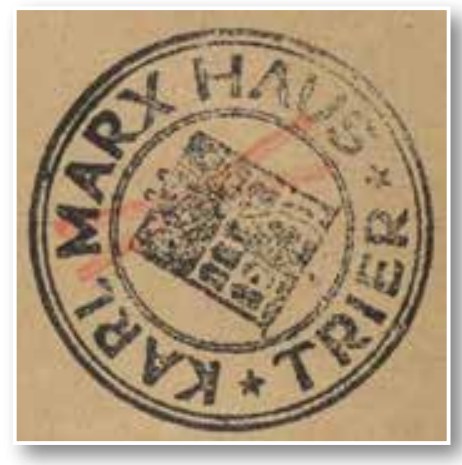

Ausgehend von den Funden freimaurerischer und marxistischer Provenienzmerkmale wurde über den Kreis der eigentlichen antiquarischen Erwerbungen hinaus noch

Abb. 6: Stempel des „Karl-Marx-Haus Trier" (in: Marx.oct.K. 40) eine thematische Recherche nach entsprechender Literatur vorgenommen, bei der noch zahlreiche weitere verdächtige Bände außerhalb des eigentlichen Untersuchungsbestands gefunden werden konnten. Zugute kam der Recherche dabei, dass es in den Beständen der Württembergischen Landesbibliothek eine alte Sachgruppe "Marxismus" gibt. Speziell in diesen Beständen fanden sich auch Bände aus den Privatbibliotheken von Gewerkschaftlern oder Arbeiterführern, so zum Beispiel aus dem Besitz des Arbeiterführers Arthur Mähr (1873-1966) aus Hof, der nach 1933 verfolgt und mehrmals verhaftet wurde. Ein anderes Beispiel aus diesem Kreis ist Willi Qualitz (1905-1938), ein Berliner Arbeiter, der in Berlin und Henningsdorf im Widerstand gegen den Nationalsozialismus aktiv war und später im spanischen Bürgerkrieg fiel. Mit seinem Namensstempel ist ebenfalls ein Buch versehen. Auch der Name von Karl Schneck (1886-1943) fand sich in einem Buch aus dem Bereich Marxismus. Schneck war SPD-Politiker in Stuttgart, 1910 wurde er Vorsitzender der SPD im Stadtbezirk StuttgartWest, 1920 wechselte er zur KPD, für die er von 1921 bis 1932 im Stuttgarter Landtag saß. Nach dem 30. Januar 1933 mehrmals verhaftet, emigrierte er 1935 in die Sowjetunion, wo er 1943 in der Verbannung starb.
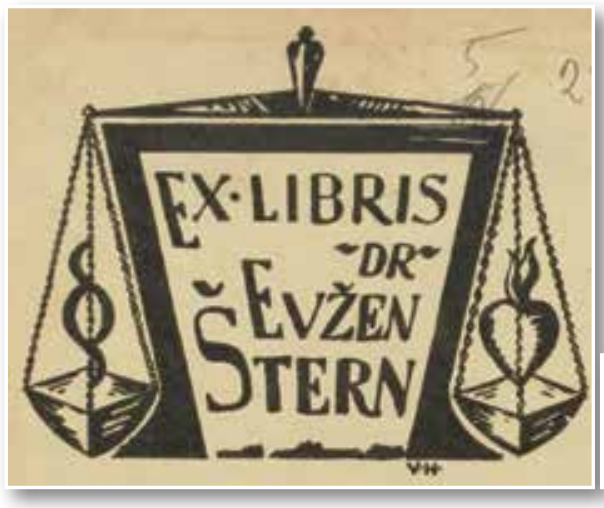

Abb. 7: Exlibris und handschriftlicher Namenszug von Evžen Štern (in: Marx.oct.647)

Ein anderer Band aus der Marxismussammlung enthält sowohl ein Exlibris des Vorbesitzers als auch dessen handschriftlichen Namenszug. Es handelt sich dabei um den tschechischen Sozialpolitiker und Publizisten Evžen Štern (1889-1942), der nach einem Jurastudium in Prag Beamter des Ministeriums für soziale Fürsorge und 1935 schließlich Direktor der Zentralen Sozialversicherungsanstalt in Prag wurde. Štern gehörte zu den Schöpfern einer modernen Sozialgesetzgebung in der Tschechoslowakei und wurde am 12. November 1942 im Konzentrationslager Mauthausen ermordet. 
Mehrere Provenienzmerkmale fanden sich von verfolgten jüdischen Buchhändlern, Antiquaren und Sammlern. Unter ihnen der Antiquar und Verleger Louis Lamm (1871-1943), von dem in einem Band ein Exlibris und ein handschriftlicher Eintrag gefunden wurden. Louis Lamm besaß eine Buchhandlung in Berlin. Bei seiner Emigration 1933 nach Amsterdam konnte er auch seinen gesamten Buchbesitz dorthin transportieren lassen. Kurz vor der Deportation im November 1943 versteckte er seine Bücher, die jedoch bald darauf entdeckt wurden. Louis Lamm wurde im Vernichtungslager Auschwitz ermordet. Ein Teil seiner Büchersammlung wurde nach dem Krieg versteigert, der größte Teil ist aber verschollen. Die Familie Lamm hatte drei Kinder, die Tochter Ruth wurde ebenfalls nach Auschwitz deportiert. Die beiden anderen Kinder emigrierten beide nach Israel, möglicherweise ergeben sich hier noch Hinweise auf Erben, denen der Band restituiert werden könnte.

Auch das jüdische Ehepaar Georg (1881-1941) und Irma Baruch (1887-1936) aus Hamburg ist sicherlich zu den Buchsammlern zu zählen.

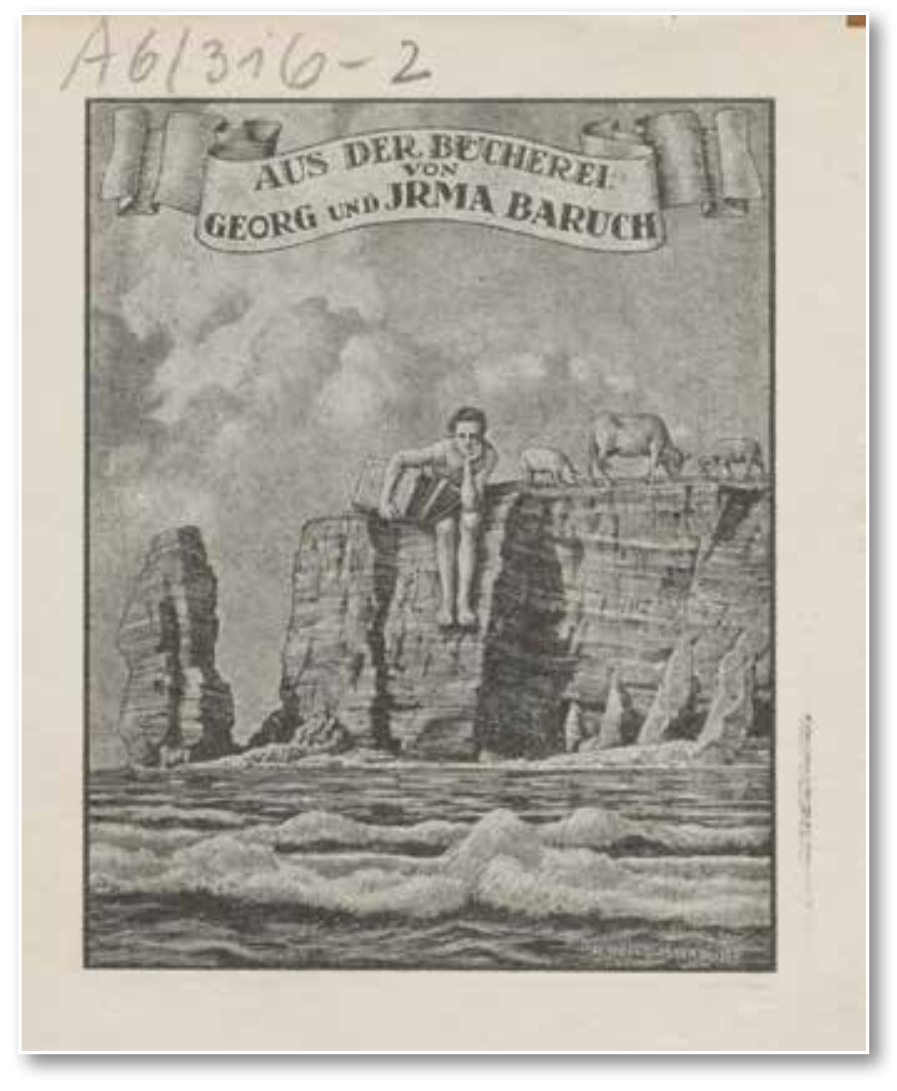

Abb. 8: Exlibris von Georg und Irma Baruch (in: A6/316-2)

In ihre Bücher klebten sie je ein Exlibris mit ihren beiden Namen, das in der Württembergischen Lan- desbibliothek bisher in insgesamt zwölf Büchern gefunden wurde. Georg Baruch war ein Hamburger Kaufmann und wurde am 8. November 1941 mit seiner Tochter Marion nach Minsk deportiert. Sein Sohn wurde in Auschwitz ermordet, nur die zweite Tochter konnte nach Israel emigrieren. Bei der Recherche nach dem Ehepaar Baruch wird deutlich, mit welch großem Nutzen die NSRaubgutforschung auf Ergebnisse der NS-OpferForschung zurückgreifen kann. Zahlreiche Erkenntnisse liefern insbesondere die in vielen deutschen Städten arbeitenden Stolperstein-Initiativen, die NS-Opfer recherchieren, ihre Lebensgeschichten erforschen und so genannte "Stolpersteine" vor den Gebäuden, in denen die Opfer zuletzt gelebt haben, verlegen. So gibt es in Hamburg auch Stolpersteine für Georg Baruch und seine beiden ermordeten Kinder. Die Geschichte der Familie ist auf der Website der Hamburger Stolperstein-Initiative (www.stolpersteine-hamburg.de) ausführlich dokumentiert. In sämtlichen in der WLB gefundenen Büchern mit dem Exlibris des Ehepaars Baruch fand sich ein weiterer Stempel des Stuttgarter Apothekers Paul Braun. Wann genau und unter welchen Umständen Paul Braun in den Besitz dieser Bücher gekommen ist, ist nicht bekannt und muss noch näher untersucht werden.

In zwei weitere Bände hat die Stuttgarter Jüdin Klara Brettheimer, geborene Lämle (1892-1944), ihren Namen eingetragen. Klara Brettheimers Mann Wilhelm (1879-1935) war Teilhaber der Firma Badisch-Württembergische Weinbrennerei Hirsch \& Lichter mit Sitz in der Bopserstraße $2 /$ Ecke Schlosserstraße. Die 1922 geborene Tochter Lisa Lotte (1922-1945) war das einzige Kind des jüdischen Ehepaares. 1930 zog die Familie in das eigene Haus an der Neuen Weinsteige 65 in Stuttgart. Klara Brettheimer und ihre Tochter gehörten zu den jüdischen Bürgern Stuttgarts, die sich am 27. November 1941 auf dem Killesberg in Stuttgart einfinden mussten. Vier Tage später, am 1. Dezember 1941, ging der erste Transport mit etwa 1.000 württembergischen Juden vom Güterbahnhof des Nordbahnhofs in Richtung Riga ab. Für die junge Lisa Lotte endet die Spur hier. Ihre Mutter Klara Brettheimer wurde am 20. Dezember 1944 im Konzentrationslager Stutthof bei Danzig ermordet. Auch hier halfen bei der Recherche wieder die Ergebnisse einer Stolperstein-Initiative weiter. Ein 
entsprechender Stein liegt heute vor dem Haus Neue Weinsteige 65 und erinnert an Klara Brettheimer, auf der entsprechenden Website sind detaillierte biographische Angaben zu ihr abrufbar (http:// www.stolpersteine-stuttgart.de).

In einem weiteren Band fand sich der handschriftliche Besitzeintrag von Hilde Adler (1885-1982), einer jüdischen Ärztin aus Stuttgart. Sie begann 1908 ihr Medizinstudium an der Universität Tübingen, 1914 erhielt sie ihre Approbation und arbeitete zunächst an der Medizinischen Universitätsklinik in Freiburg. 1913 heiratete sie Jakob Adler, eine gemeinsame Tochter wurde 1917 geboren. Im gleichen Jahr zog die Familie nach Stuttgart. Unmittelbar nach der "Machtergreifung" entschloss sie sich zur Emigration und floh 1934 zunächst nach Südamerika, später in die USA. Um die Flucht zu finanzieren, musste der gesamte Besitz verkauft werden, darunter wahrscheinlich auch die Bücher. Generell gelten auch unter Zwang - und meist weit unter Wert - verkaufte Besitztümer als unrechtmäßig enteignetes Eigentum, das restituiert werden muss.

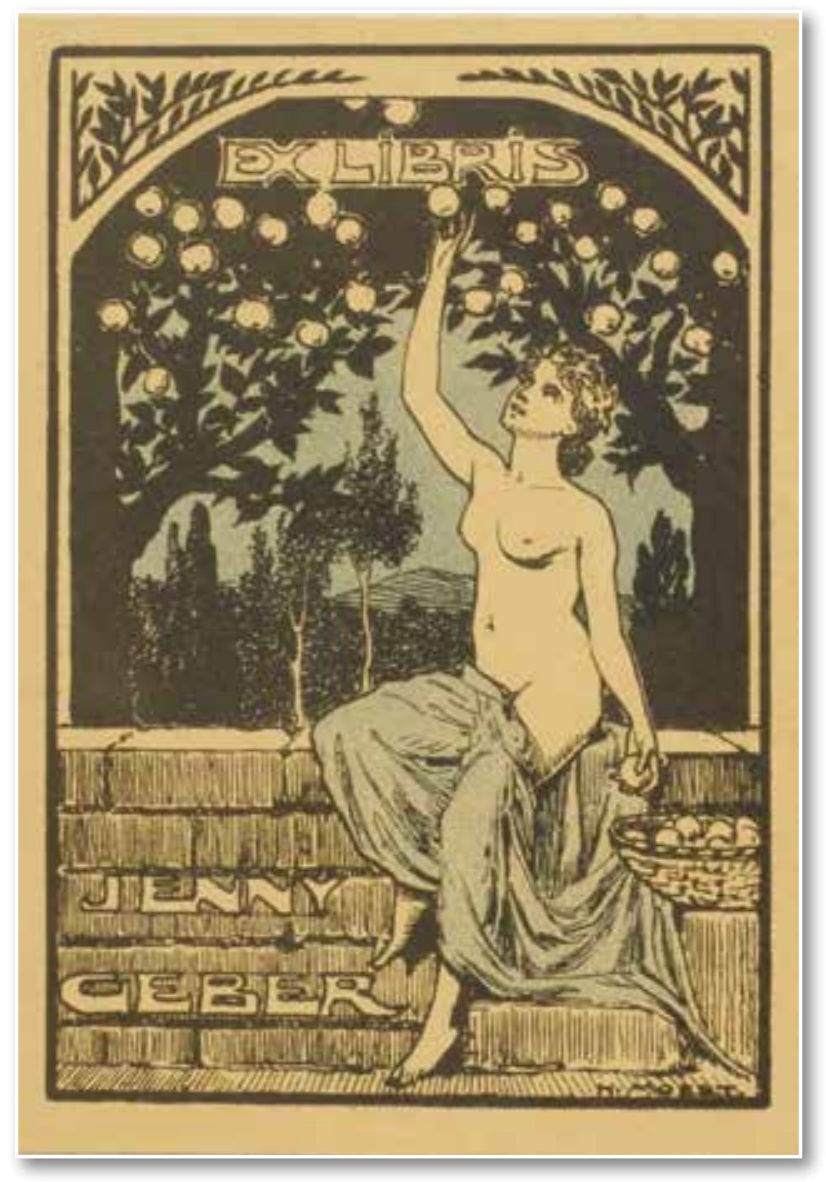

Abb. 9: Exlibris von Jenny Geber (in: A6/818)
Um einen etwas komplizierteren Fall handelt es sich bei dem Exlibris von Jenny Geber (1873-1943), das bisher in einem Buch entdeckt wurde. Jenny Gebers Name findet sich in einem für die NSRaubgut-Forschung zentralen Verzeichnis von NS-Opfern, dem so genannten "Gedenkbuch" des Bundesarchivs (https://www.bundesarchiv.de/ gedenkbuch). Jenny Geber, geborene Benfey, ist demnach am 28. Januar 1943 in Theresienstadt gestorben.

Ob es sich dabei aber um die gesuchte Jenny Geber handelt, bleibt zunächst unklar. Der Verdacht erhärtet sich jedoch über den Mädchennamen Benfey, denn der Künstler, der das Exlibris von Jenny Geber gestaltet hat, heiratete eine geborene Benfey, die wie Jenny Geber ebenfalls aus Hannover stammte. Möglicherweise handelt es sich also um Verwandte. Weitere Angaben zu Jenny Geber ließen sich aus alten Zeitungen gewinnen, auch dieses ein Rechercheinstrument, das in der NS-Raubgutforschung gewinnbringend eingesetzt werden kann. Dabei nimmt das Angebot an digitalisierten historischen Zeitungen immer mehr zu, und immer häufiger sind diese auch als Volltext durchsuchbar. Entsprechende Angebote bieten beispielsweise das österreichische Zeitungsportal ANNO (http://anno.onb.ac.at) sowie das europaweite Portal Europeana Newspapers (http://www. theeuropeanlibrary.org/tel4/newspapers). Auf diesem Weg konnte in einer Berliner Tageszeitung die Todesanzeige der Schwiegermutter von Jenny Geber vom 15. Mai 1912 entdeckt werden, in der als weiterer Name Hedwig Geber erscheint. Eine Anfrage beim Centrum Judaicum in Berlin ergab, dass Jenny Geber eine Tochter hatte, die sich wohl während des Krieges in Berlin verstecken konnte und 1960 in Starnberg starb. Sie hatte vermutlich keine Kinder.

Darüber hinaus konnten Besitzmerkmale von zwei weiteren Opfern der Judenverfolgung in der Zeit des Nationalsozialismus gefunden werden. Ein Exlibris in einem Band verweist auf Dr. Otto Rothschild (1872-1940), einen jüdischen Arzt aus Frankfurt am Main, der 1940 zusammen mit seiner Ehefrau Selbstmord beging, um der nationalsozialistischen Verfolgung zu entgehen. In einem weiteren Band gibt es einen Besitzeintrag des Rabbiners Israel Nobel (1887-1962) aus Schneidemühl, der 
1925 nach Berlin ging, 1936 dann nach Palästina emigrierte und 1962 in Jerusalem starb.

Neben diesen Provenienzmerkmalen von jüdischen Vorbesitzern fanden sich einzelne Bände mit Besitzvermerken von verfolgten Einzelpersonen, die dem Widerstand gegen den Nationalsozialismus zuzurechnen sind. Dies ist der Fall bei Margarete Klinckerfuß (1877-1959), einer in Stuttgart geborenen Pianistin, die sich nach 1933 mehrfach kritisch über den Nationalsozialismus geäußert hatte und deshalb 1937 von der Gestapo verhaftet und in die psychiatrische Klinik Christophsbad in Göppingen eingewiesen wurde. Nach 1945 arbeitete sie dort als Musiktherapeutin.

Ein heute nur noch schwach lesbarer handschriftlicher Namenseintrag in einem gefundenen Buch stammt wahrscheinlich von der 1944 in BerlinPlötzensee hingerichteten Widerstandskämpferin Elisabeth von Thadden (1890-1944).

\section{Ausblick}

Die Förderung des Projekts zur Suche nach NSRaubgut in den Beständen der Württembergischen Landesbibliothek ist vom Deutschen Zentrum Kulturgutverluste von zunächst einem auf jetzt drei Jahre Projektlaufzeit verlängert worden. In dieser Zeit sollen insgesamt ca. 60.000 Bände durchgesehen und entsprechende verdächtige Provenienzmerkmale verzeichnet werden. Parallel werden die Nachforschungen nach Opfern und deren Nachkommen intensiviert. Wie oben erwähnt, sollen dann auch Stücke, die als NS-Raubgut identifiziert werden konnten, an die Erben der Opfer restituiert werden.

Die Ergebnisse des Projekts werden sowohl in einer Publikation als auch in einer Ausstellung spätestens zum Projektende 2019 umfassend veröffentlicht. Näheres und Aktuelles über das Projekt sowie die neuesten Ergebnisse finden sich auch unter: http://www.wlb-stuttgart.de/die-wlb/ ns-raubgutforschung

Maria Nüchter / Hans-Christian Pust 